\title{
HMGA2 regulates epithelial-mesenchymal transition and the acquisition of tumor stem cell properties through TWIST1 in gastric cancer
}

\author{
WEI LI, ZIWEI WANG, LANG ZHA, DEQUAN KONG, GANG LIAO and HUI LI \\ Department of Gastrointestinal Surgery, First Affiliated Hospital of Chongqing Medical University, \\ Chongqing 400016, P.R. China
}

Received July 5, 2016; Accepted August 6, 2016

DOI: $10.3892 /$ or.2016.5255

\begin{abstract}
High expression of high mobility group protein A2 (HMGA2) is correlated with the invasiveness of gastric cancer and is an independent prognostic factor. The reason may be that HMGA2 promotes epithelial-mesenchymal transition (EMT) and the acquisition of tumor stem cell properties, yet the mechanism remains unclear. In this study, immunohistochemistry and western blot analysis revealed that the expression of HMGA2 and Twist-related protein 1 (TWIST1) in gastric carcinoma tissues was higher than that in the peritumoral tissues and that the expression levels of these two proteins were positively correlated. The protein expression levels of HMGA2 and TWIST1 were high in the poorly differentiated gastric cancer MKN-45 cells and were low in the moderately differentiated SGC-7901 cells. TWIST1 was inhibited after HMGA2 interference and was significantly increased after overexpression of HMGA2. Luciferase experiments showed that TWIST1 was a direct downstream target gene of HMGA2. The simultaneous interference of HMGA2 expression and the overexpression of TWIST1 in MKN-45 cells reversed the inhibitory effect of HMGA2 interference on the invasion and migration of gastric cancer cells, EMT and the expression of stemness markers. However, the simultaneous overexpression of HMGA2 and the interference of TWIST1 expression in the SGC-7901 cells reversed the promoter effect of HMGA2 overexpression on the invasiveness and migration of gastric cancer cells, EMT and the expression of stemness markers. In addition, animal experiments showed that TWIST1 overexpression reversed the inhibition of HMGA2 interference on the metastasis of MKN-45 cells. Therefore, HMGA2 regulates the EMT of gastric cancer cells and the acquisition of tumor stem cell
\end{abstract}

Correspondence to: Dr Ziwei Wang, Department of Gastrointestinal Surgery, First Affiliated Hospital of Chongqing Medical University, Chongqing 400016, P.R. China

E-mail: wangziwei571@sina.com

Key words: HMGA2, TWIST1, gastric cancer, epithelialmesenchymal transition, cancer stem cells, stemness properties through direct regulation of the downstream target gene TWIST1.

\section{Introduction}

Gastric cancer is one of the most common gastrointestinal tumors. Although its mechanism of development is unclear, the invasive behavior and metastasis of cancer cells are the main cause of death in patients with gastric cancer. In recent years, many studies have suggested that epithelial-mesenchymal transition (EMT) in epithelial tumors such as gastric cancer is an important step in tumor metastasis (1). During the EMT process, cell invasiveness and migration are enhanced, and tumor cells acquire stem cell-like characteristics, which promote tumor invasion and metastasis (2-4).

High mobility group protein A2 (HMGA2) is a non-histone chromosomal protein, which plays a structural role as a transcription factor primarily by binding the AT-rich regions in the DNA sequence of target genes; this protein thus affects embryogenesis, tissue development and tumorigenesis (5). In recent years, HMGA2 has been reported to be highly expressed in tissues derived from thyroid (6), colon (7) and gastric cancer (5). It has also been reported that HMGA2 can induce tumor cells to undergo EMT via interference of the cell cycle (8-11), that HMGA 2 can help tumor cells acquire stem cell properties, and that it can promote tumor metastasis (12). Recent studies have demonstrated that HMGA2 may promote EMT in gastric cancer (13) and promote an increase in the expression of several tumor markers involved in breast cancer (14). HMGA2 serves as a specific downstream target of Lin28b, which regulates the self-renewal of mouse hematopoietic stem cells (15). HMGA2 has been suggested to mediate breast tumor metastasis via the promotion of the expression of LOX and syndecan-2 (16). Previous studies, including ours, have shown that HMGA2 may be responsible for the invasiveness and metastasis of gastric cancer and for its poor prognosis $(5,13,17)$, yet the related mechanism remains unclear.

Recently, TWIST proteins have been re-established as important transcription factors associated with EMT $(18,19)$; Twist-related protein 1 (TWIST1) is most closely related to tumor metastasis and angiogenic mimicry formation. Some studies have reported that the potential combination 
of TWIST1 and E-box, which is an E-cadherin promoter, negatively regulates the transcription of the E-cadherin gene. This in turn induces EMT and the acquisition of tumor stem cell properties, which enhances invasiveness, metastasis and the angiogenic capacity of breast cancer cells (20-22). One study revealed that TWIST1 enhanced cell migration beyond EMT, as TWIST1 induced the activation of Rac1 (23). Yang et al (24) confirmed that TWIST1 may be incorporated into the intron region of the BMI1 gene and that it can generate a stem cell-like phenotype and promote tumor initiation. TWIST1 overexpression and poor tumor prognosis are associated with a high rate of tumor invasion and metastasis (25). Research has shown that TWIST1 is involved in the malignant progression of colorectal cancer (26), but no evidence has indicated its involvement in gastric cancer.

Our previous study found that HMGA2 induced EMT in gastric cancer cells and that this phenomenon was related to the activation of the Wnt/ $\beta$-catenin signaling pathway. Using ChIP-on-chip by chromatin immunoprecipitation (ChIP) technology, we also screened TWIST genes that may be associated with EMT in gastric cancer cells $(13,27)$. We speculated that HMGA2 may regulate EMT and the acquisition of tumor stem cell properties via the regulation of the downstream target gene TWIST1. Therefore, the aim of the present study was to investigate the relationship between HMGA2 and TWIST1 in gastric cancer and to determine whether HMGA2 can regulate EMT in gastric cancer cells, as well as the acquisition of tumor stem cell properties, through the regulation of TWIST1.

\section{Materials and methods}

Ethics statement. This study was approved by the Medical Ethics Review Committee of the First Affiliated Hospital of Chongqing Medical University. All of the procedures that involved animals were conducted as indicated in the Guidelines of the National Institutes of Health (NIH) for Animal Care (Guide for the Care and Use of Laboratory Animals, Department of Health and Human Services, NIH Publication no. 86-23, revised 1985). We made every effort to minimize suffering and to minimize the number of animals used.

Patient collection. In total, 172 paraffin-embedded tissue specimens (142 gastric cancer tissues and 30 peritumoral tissues) were collected from the archives of the Department of Pathology, Chongqing Medical University. These tissue samples were subjected to the avidin-biotin-peroxidase method for immunohistochemical analysis. An additional 72 fresh surgical specimens (36 gastric cancer tissues and corresponding peritumoral tissues) were obtained from the Department of Gastrointestinal Surgery, First Affiliated Hospital of Chongqing Medical University. All specimens were frozen in liquid nitrogen immediately after surgical resection and were maintained at $-80^{\circ} \mathrm{C}$ until protein extraction was performed. None of the patients had received preoperative treatment, such as radiation or neoadjuvant chemotherapy.

Immunohistochemical analysis. Tissue sections were deparaffinized in two changes of xylene, and antigen retrieval was performed by heating the sections in $0.01 \mathrm{M}$ sodium citrate buffer in a microwave oven at $95^{\circ} \mathrm{C}$ for $15 \mathrm{~min}$. The slides were incubated in $3 \%$ hydrogen peroxide for $15 \mathrm{~min}$ and were then incubated in $0.5 \%$ Triton-X-100 (Beyotime Biotech, Jiangsu, China) for $15 \mathrm{~min}$. The slides were blocked with 5\% BSA for 30 min and were incubated with rabbit primary antibodies against human HMGA2 (1:100; Cell Signaling Technology, Danvers, MA, USA) and TWIST1 (1:100, ProteinTech Group, Inc., Wuhan, China) overnight at $4^{\circ} \mathrm{C}$. Negative controls were treated identically but were not incubated with the primary antibodies. Tissue sections were washed in PBS ( $3 \times 5 \mathrm{~min})$, incubated with the appropriate secondary antibody for $30 \mathrm{~min}$, washed in PBS ( $3 \times 5 \mathrm{~min})$ and incubated for $30 \mathrm{~min}$ with streptavidin-HRP (Beijing Zhongshan Jinqiao Biotechnology Co., Ltd., Beijing, China). Immunoreactivity was visualized using a DAB kit (Beijing Zhongshan Jinqiao Biotechnology Co., Ltd.); the slides were counterstained with hematoxylin and mounted with PVP. The percentage of positive cells was scored according to the following criteria: $0,<10 \% ; 1,10-30 \%$; $2,>30-50 \%$; and $3,>50 \%$ (28). The patients were subsequently categorized into either a positive expression group (score of 1-3) or a negative expression group (score of 0 ).

Cell culture. MKN-45, MKN-28 and SGC-7901 human gastric carcinoma cell lines were obtained from the Key Laboratory of General Surgery at the First Affiliated Hospital of Chongqing Medical University. The GES-1 human immortalized gastric epithelial cell line was purchased from the Chinese Type Culture Collection. The cell lines were cultured in RPMI-1640 medium (Hyclone, Shanghai, China) supplemented with $10 \%$ fetal bovine serum (FBS) (Hyclone) and 2\% penicillin-streptomycin (Beyotime) in a humidified atmosphere of $95 \%$ air and $5 \% \mathrm{CO}_{2}$ at $37^{\circ} \mathrm{C}$. The cells were cultured until a confluence of $80 \%$ was reached, at which point they were passaged by trypsinization.

Western blot analysis. The cells were lysed in buffer containing $1 \% \mathrm{NP} 40,1 \mathrm{mmol} / \mathrm{l}$ EDTA, $50 \mathrm{mmol} / 1$ Tris- $\mathrm{HCl}$ (pH 7.5), and $150 \mathrm{mmol} / \mathrm{l} \mathrm{NaCl}$ supplemented with a complete protease inhibitor mixture (Sangon Biotech, Shanghai, China). Total proteins were separated by SDS-PAGE and transferred to PVDF membranes (Millipore, Billerica, MA, USA). The membranes were then blocked in 5\% non-fat milk in TBS for $2 \mathrm{~h}$ and probed with antibodies to HMGA2 (1:1,000; Cell Signaling Technology), TWIST1 (1:1,000; Proteintech Group), E-cadherin (1:100; Santa Cruz Biotechnology, Inc., Santa Cruz, CA, USA), N-cadherin (1:1,000) and vimentin $(1: 1,000$; both from eBioscience, Inc., San Diego, CA, USA), Oct4 (1:1,000; San Ying Biotechnology, Wuhan, China), CD44 (1:1,000; San Ying Biotechnology) and GAPDH (1:500; Millipore). After the membranes were washed, they were incubated with the appropriate secondary antibodies for $1 \mathrm{~h}$ at $37^{\circ} \mathrm{C}$. The results were visualized using an enhanced chemiluminescence kit (Beyotime Biotech). Each band was quantified using ImageJ software and normalized to GAPDH (Beyotime Biotech).

Cell transfection. Human HMGA2 and TWIST1 cDNAs were cloned into pLV-UbC-IRES2-EGFP via homologous recombination [S\&E (Shanghai) Bio-pharmaceutical, Shanghai, China]. The HMGA2 shRNA sequences, the TWIST1 shRNA sequences and the scrambled RNA were synthesized by Ribobio 
Corporation (Guangzhou, China). The TWIST1 shRNA sequences and the scrambled RNA were cloned into the hU6-Neomycin Vector by S\&E (Shanghai) Bio-pharmaceutical. The cells were transiently transfected with TWIST1 shRNA or scrambled RNA (con) using Lipofectamine 2000 (Invitrogen Life Technologies, Shanghai, China) according to the manufacturer's instructions. The TWIST1 overexpression vector was purchased from S\&E (Shanghai) Bio-pharmaceutical. The sequences of the HMGA2- and TWIST1-specific shRNAs used in these experiments were as follows: control-shRNA, 5'-GAC GAGCGGCACGTGCACATT-3'; HMGA2-shRNA, 5'-CGGC CAAGAGGCAGACCTATT-3'; TWIST1-shRNA, 5'-CGGAC AAGCTGAGCAAGATTC-3'. The cells were plated in 6-well clusters and transfected for $24 \mathrm{~h}$ or $48 \mathrm{~h}$. Transfected cells were used in further assays or for RNA/protein extraction.

Luciferase reporter assay. The ChIP-on-chip experiments showed that HMGA2 could bind to the 5650-5900 bp region before the TWIST1 transcriptional start site; therefore, this region was amplified by $\mathrm{PCR}$ and inserted into the pGL3-Promoter luciferase reporter vector (Promega, Madison, WI, USA). All constructs were verified by DNA sequencing. SGC-7901 cells were plated in 96-well clusters and then co-transfected with 100-ng constructs with or without the HMGA2 overexpression plasmid. At $48 \mathrm{~h}$ after transfection, the luciferase activity was detected using a dual-luciferase reporter assay system (Promega) and was normalized to Renilla luciferase activity. All primers used were purchased from Genewiz Biotechnologies, Inc. (Suzhou, China) and are as follows: forward, 5'-TCTATCGATAGGTACCAGGAAAA ATACAGTCTGACTTC-3' and reverse, 5'-GATCGCAGATC TCGAGTTTCTGTAATTGTTGCCCAAG-3'.

Wound healing assay. HepG2 and SMMC7721 cells were transfected with the following: HMGA2 shRNA or HMGA2 overexpression vectors alone; HMGA2 shRNA combined with the TWIST1 overexpression clone; or the HMGA2 overexpression clone with TWIST1 shRNA. The transfected cells were seeded in 6-well plates and allowed to grow to $100 \%$ confluence; afterwards, a scratch was made across the cell monolayer. The cells were rinsed with PBS and fresh growth medium was added. The cells were incubated for $24 \mathrm{~h}$ and then imaged using a phase-contrast microscope.

Cell invasion assay. For the cell invasion assay, $1 \times 10^{4}$ cells were seeded on Matrigel-coated Transwell cell-culture inserts (Invitrogen Life Technologies, Carlsbad, CA, USA) with RPMI-1640 medium with 2\% FBS. The bottom chamber was filled with $600 \mu 1$ RPMI-1640 medium with $10 \%$ FBS. After $48 \mathrm{~h}$ of incubation, cells on the lower surface of the membrane were stained with crystal violet. The number of cells was counted in three random fields under a light microscope.

Animal experiment. Female BALB/c mice at 6 weeks of age were obtained from the National Biological Industry Base, Laboratory Animal Center of Chongqing Medical University. The mice were randomly divided into four groups (five mice per group) and were maintained under pathogen-free conditions. The mice were intravenously injected with MKN-45 cells, and the mice were observed using an imaging system
Table I. Relationship between the protein expression of HMGA2 and TWIST1 by immunohistochemistry.

\begin{tabular}{lccccc}
\hline & \multicolumn{2}{c}{ TWIST1 expression } & & & \\
\cline { 2 - 3 } & High & Low & Total & $\chi^{2}$ & P-value \\
\hline HMGA2 & & & & & \\
expression & & & & & \\
High & 40 & 23 & 63 & 29.254 & $<0.001$ \\
Low & 15 & 64 & 79 & & \\
Total & 55 & 87 & 142 & & \\
\hline
\end{tabular}

at one and two weeks after inoculation. The mice were then dissected four weeks after inoculation to observe MKN-45 cell metastasis.

Statistical analysis. All experimental data are presented as the means $\pm \mathrm{SD}$, and single comparisons between two groups were evaluated by Student's t-test using SPSS 20.0. Associations between the expression levels of HMGA2 and TWIST1 were analyzed by the Pearson correlation coefficient. $\mathrm{P}<0.05$ was considered statistically significant.

\section{Results}

Protein expression of HMGA2 and TWIST1 is high in gastric cancer according to immunohistochemistry and western blot analysis. To investigate the association between HMGA2 and TWIST1 expression in gastric cancer, we performed immunohistochemical staining for these proteins in tissue specimens from 142 gastric cancer patients. The staining for HMGA 2 and TWIST1 in representative clinical samples is shown in Fig. 1A. Among the 142 gastric cancer specimens, 75 $(52.8 \%)$ and $65(45.8 \%)$ cases were positive for HMGA2 and TWIST1 expression, respectively. In addition, immunohistochemical analysis showed that the expression of HMGA2 and that of TWIST1 were positively correlated $(\mathrm{P}<0.01$; Table I). HMGA2 and TWIST1 were largely expressed in the nucleus of gastric cancer cells. The expression levels of HMGA2 and TWIST1 were significantly higher in gastric cancer tissues compared with those in the peritumoral tissues $(\mathrm{P}<0.01)$. Additionally, we tested the protein expression of HMGA2 and TWIST1 in 36 paired tissue samples via western blot analysis. As expected, the protein expression levels of HMGA2 and TWIST1 were significantly higher in the gastric cancer tissues than these levels the in peritumoral tissues $(\mathrm{P}<0.05$; Fig. 1B).

Expression of HMGA2 and TWIST1 in gastric cancer cell lines. To investigate whether HMGA2 and TWIST1 levels are also increased in gastric cancer cell lines compared with immortalized gastric epithelial cells, we assessed the HMGA2 and TWIST1 protein levels in the human gastric cancer cell lines MKN-45, MKN-28, and SGC-7901, as well as in the human immortalized gastric epithelial cell line GES-1, by western blot analysis. The HMGA2 and TWIST1 protein levels were significantly higher in the poorly differentiated gastric cancer cell line (MKN-45), the moderately differentiated cell 
A

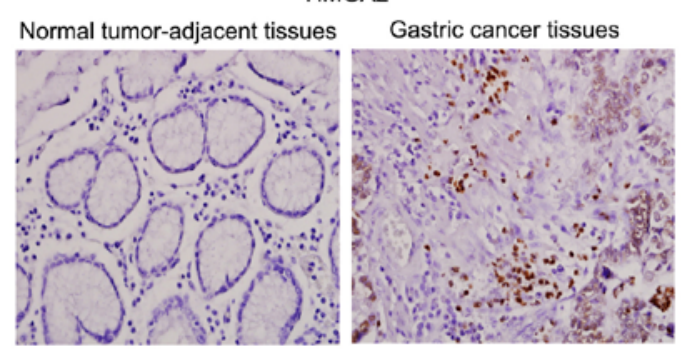

B

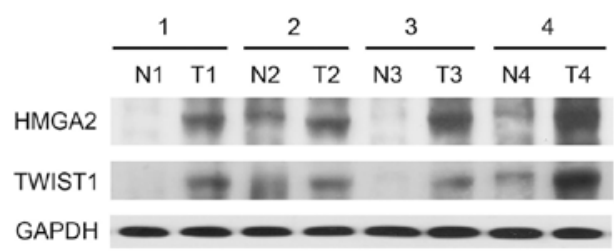

TWIST1
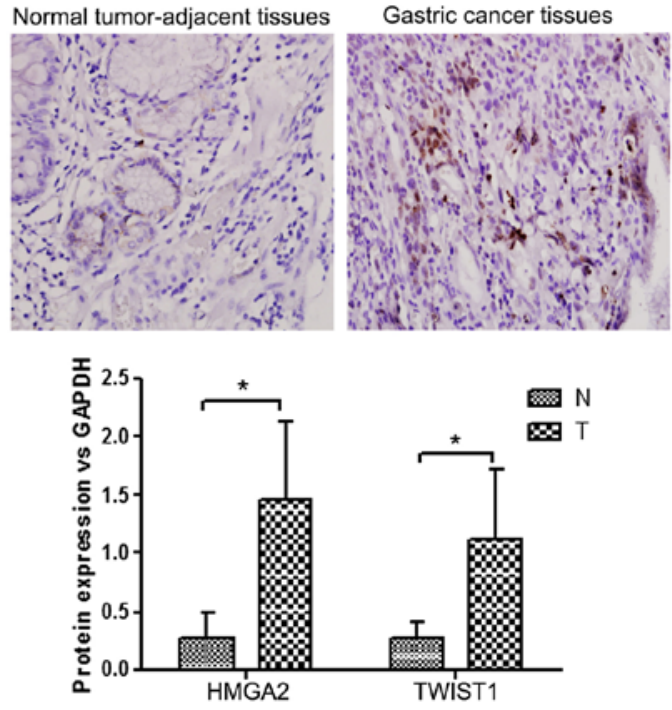

C
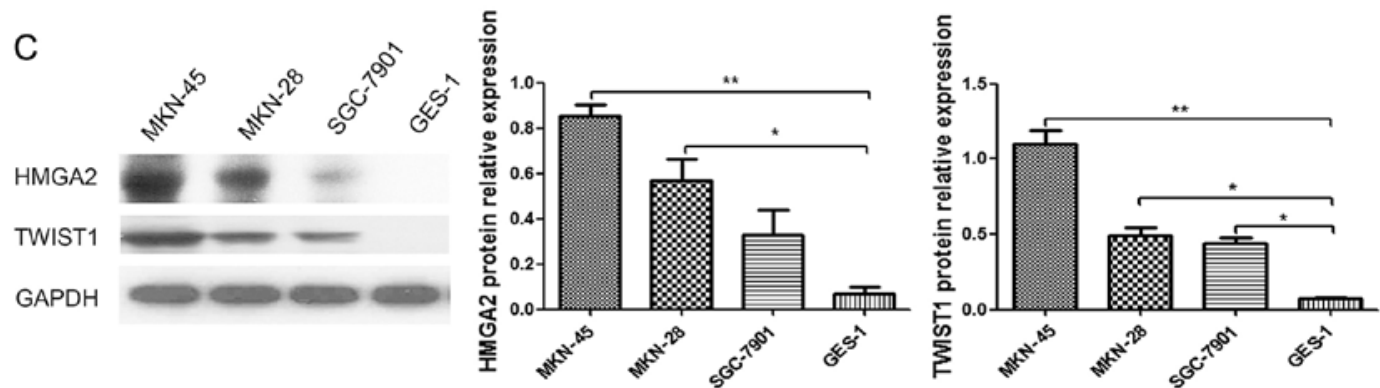

Figure 1. HMGA2 and TWIST1 were highly expressed in gastric cancer tissues and gastric cancer cells. (A) Expression of HMGA2 and TWIST1 in gastric cancer tissues and adjacent normal tissues was detected by immunohistochemistry. (B) Expression of HMGA2 and TWIST1 in gastric cancer tissues and normal adjacent tissues was detected by western blot analysis. N, adjacent normal tissues; T, tumor tissues; 10 gastric cancer tissues were randomly tested. (C) Expression of HMGA2 and TWIST1 was detected by western blot analysis in the gastric cancer cell lines MKN-45, MKN-28, and SGC-7901 and in the normal gastric cell line GES-1. The assay was repeated three times. ${ }^{*} \mathrm{P}<0.05,{ }^{* *} \mathrm{P}<0.01$. HMGA2, high mobility group protein $\mathrm{A} 2$.

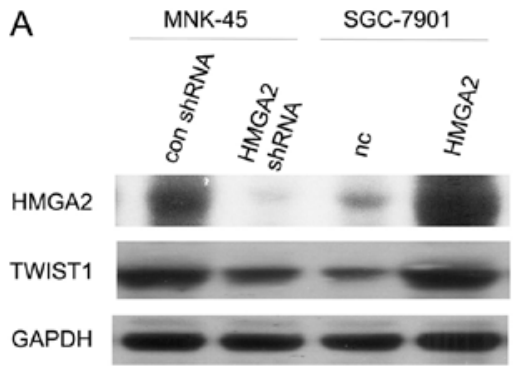

B

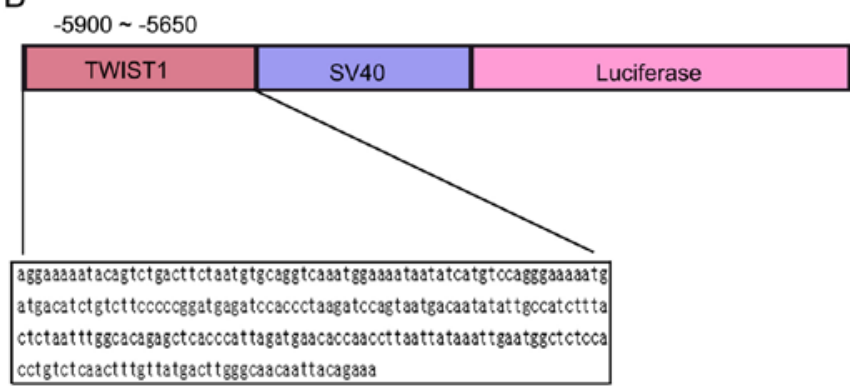

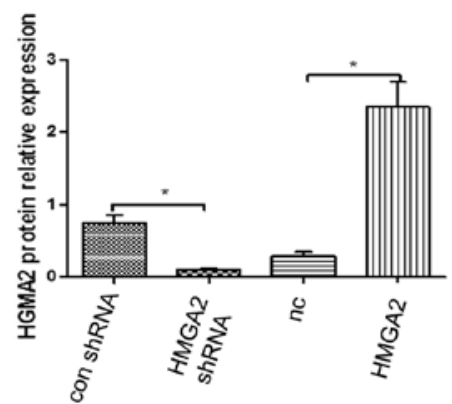
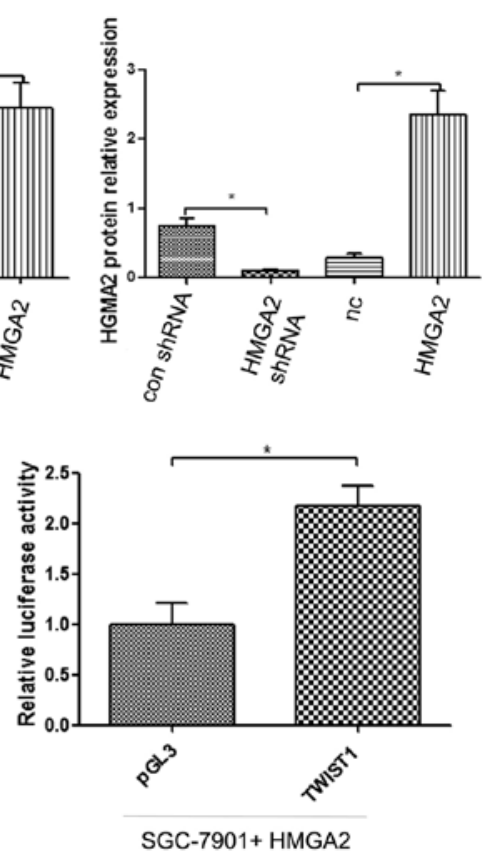

Figure 2. Expression of HMGA2 is regulated by the upstream sequence in the TWIST1 coding region. (A) HMGA2 expression was inhibited in MKN-45 cells, and HMGA2 was overexpressed in SGC-7901 cells. The protein expression of HMGA2 and TWIST1 was then detected by western blot analysis. The experiment was repeated three times. (B) Construction of the luciferase reporter vector plasmids of the upstream sequence of the TWIST1 coding region, the co-transfection of SGC-7901 cells with the HMGA2 expression plasmid, and the detection of the luciferase activity of the reporter vector. The experiment was repeated three times. "P<0.05. HMGA2, high mobility group protein $\mathrm{A} 2$. 
A
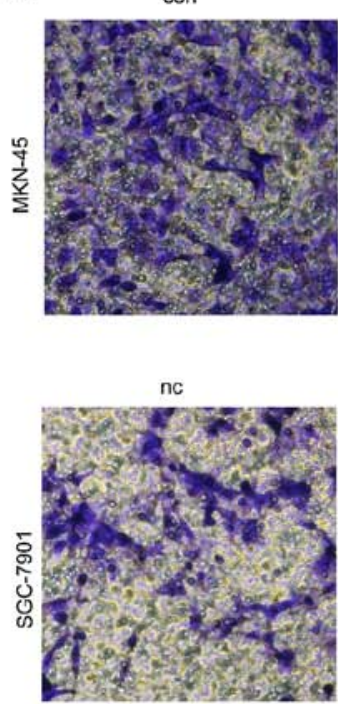

B
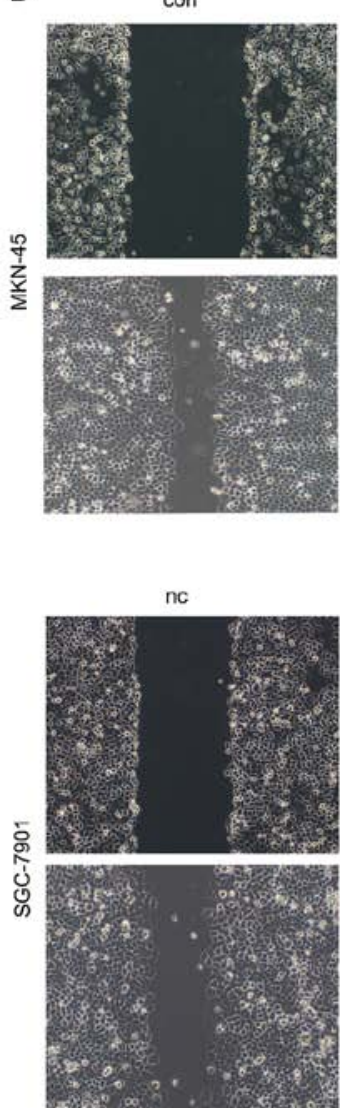

HMGA2 ShRNA

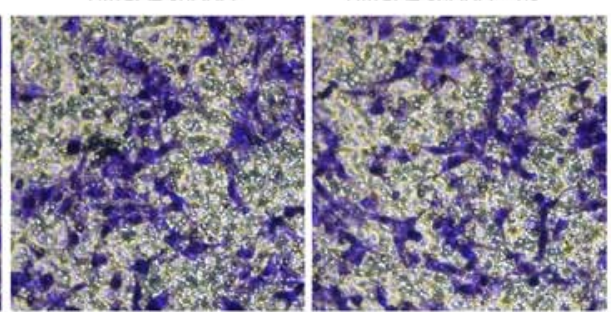

HMGA2

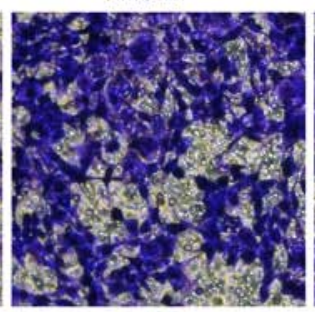

HMGA2 + con

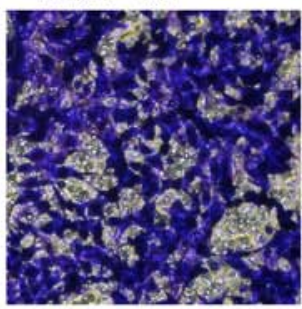

HMGA2 SHRNA + TWIST1

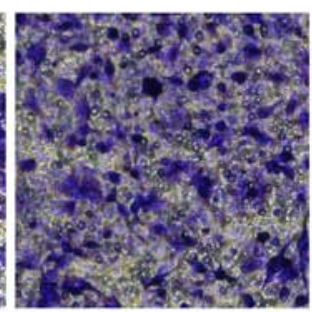

HMGA2 + TWIST1 1 ShRNA

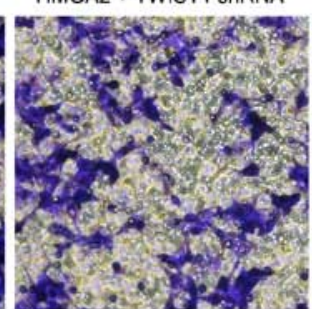

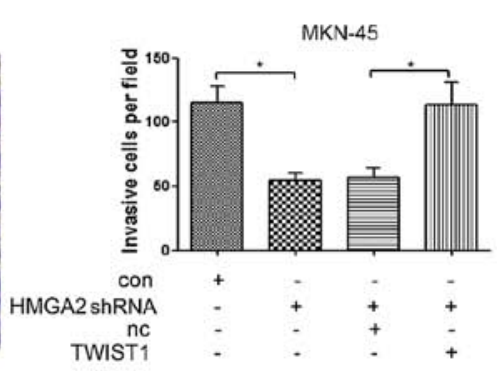

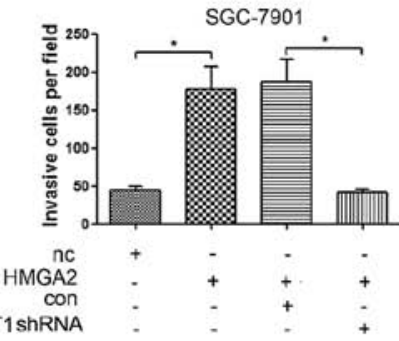

TWIST1 1shRNA

HMGA2 ShRNA + TWIST1
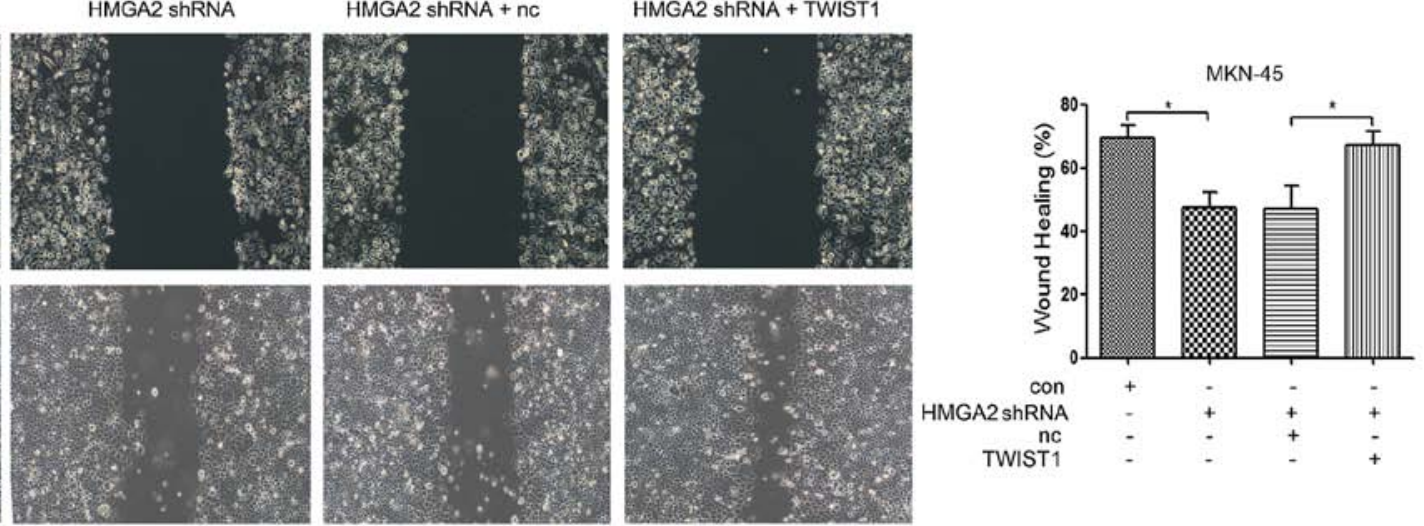

Figure 3. HMGA2 affects the invasion and migration of gastric cancer cells via TWIST1. (A) MKN-45 cells were transfected with con, HMGA2 shRNA, HMGA2 shRNA + NC, or HMGA2 shRNA + TWIST1. Then, a Transwell assay was used to detect cell invasiveness in each group; SGC-7901 cells were transfected with NC, HMGA2, HMGA2 + con, or HMGA2 + TWIST1 shRNA. Then, a Transwell invasion assay was used to detect cell invasiveness in each group. (B) MKN-45 cells were transfected with con, HMGA2 shRNA, HMGA2 shRNA + NC, or HMGA2 shRNA + TWIST1; and then a scratch assay was used to detect cell migration in each group. SGC-7901 cells were transfected with NC, HMGA2, HMGA2 + con, or HMGA2 + TWIST1 shRNA; and then a scratch assay was used to detect cell migration in each group. ${ }^{*} \mathrm{P}<0.05,{ }^{* *} \mathrm{P}<0.01$. HMGA2, high mobility group protein $\mathrm{A} 2$.

line (SGC-7901), and the well-differentiated cell line (MKN28) compared with the GES-1 cells $(\mathrm{P}<0.01$; Fig. $1 \mathrm{C})$.

TWIST1 is a direct target of HMGA2 in gastric cancer cells. HMGA2 and TWIST1 protein expression levels in various gastric epithelial cell lines such as MKN-45-con
shRNA, MKN-45-HMGA2 shRNA, SGC-7901-NC, and SGC-7901, in which HMGA2 was originally overexpressed, were detected by western blot analysis. It was found that after the application of HMGA2 shRNA in the MKN-45 cells, the expression levels of HMGA2 and TWIST1 were significantly decreased ( $\mathrm{P}<0.05$; Fig. $2 \mathrm{~A})$. After HMGA2 


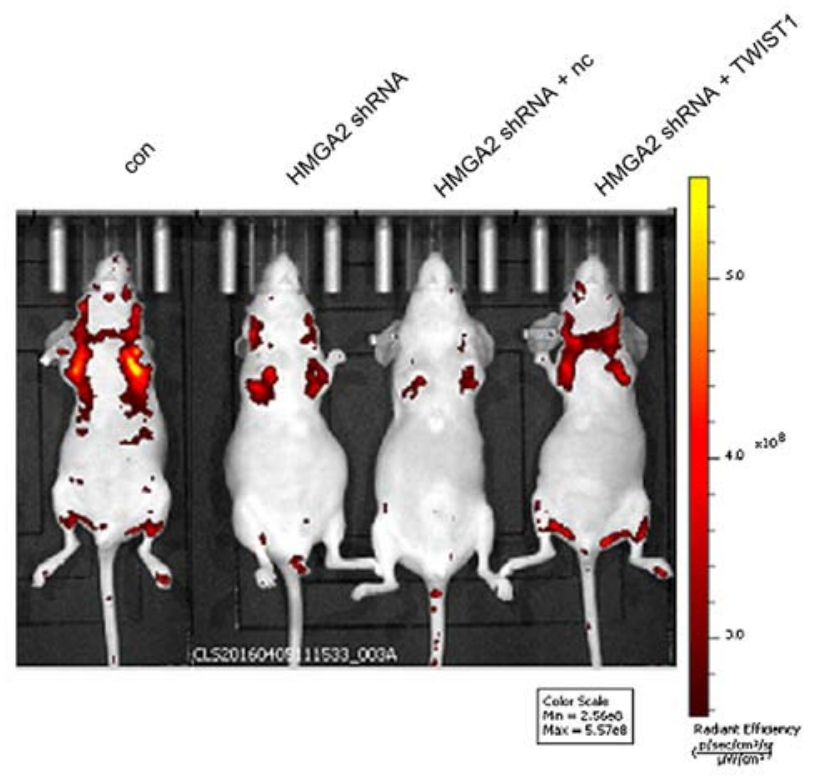

Figure 4. HMGA2 affects the in vivo metastasis of gastric cancer cells via TWIST1. MKN-45 cells were transfected with con, HMGA2 shRNA, HMGA2 shRNA + NC, or HMGA2 shRNA + TWIST1; and then stable strains were screened. After the cells were cultured and expanded, the cells at a concentration of $5 \times 10^{6}$ cells $/ \mathrm{ml}$ (volume, $0.1 \mathrm{ml}$ ) were then injected into the tail veins of mice. In vivo imaging was performed after 4 weeks. HMGA2, high mobility group protein A2.

was overexpressed in the SGC-7901 cells, the expression levels of TWIST1 and HMGA2 were significantly increased $(\mathrm{P}<0.05$; Fig. 2A). Simultaneously, we co-transfected the pGL3-TWIST1-Promoter vector and the HMGA2 expression plasmid or the negative control (NC) into SGC-7901 cells. The luciferase activity of the HMGA2-overexpressing plasmid-transfected cells was significantly increased compared with that of the NC-transfected cells (Fig. 2B).

HMGA2 regulates the invasion and migration of gastric cancer cells via TWIST1. The inhibition of HMGA2 suppressed the invasion and migration of MKN-45 cells, and the simultaneous overexpression of TWIST1 reversed the inhibitory effect of HMGA2 expression on the invasion and migration of gastric cancer cells (Fig. 3A and B). The overexpression of HMGA2 significantly promoted the invasion and migration of SGC-7901 cells, whereas the simultaneous inhibition of TWIST1 expression reversed the promotive effect of HMGA2 overexpression on the invasion and migration of gastric cancer cells (Fig. 3A and B).

Overexpression of TWIST1 reverses the inhibitory effect of HMGA2 abrogation on the metastasis of gastric cancer cells in nude mice. To further investigate whether HMGA2 regulates the invasion and migration of gastric cancer cells via TWIST1, we injected MKN-45 cells into the tail veins of mice to detect the in vivo metastasis of MKN-45 cells after the application of HMGA2 shRNA. The results showed that the metastatic ability of MKN-45 cells was significantly decreased after the abrogation of HMGA2 expression, whereas the simultaneous overexpression of TWIST1 reversed the inhibitory effect of HMGA2 interference on the metastasis of gastric cancer cells in nude mice (Fig. 4).
HMGA2 regulates EMT and the acquisition of stem cell properties in gastric cancer cells via TWIST1. The interference of HMGA2 expression notably inhibited EMT of MKN-45 cells and the expression of the stemness markers CD44 and OCT4. However, at the same time, the overexpression of TWIST1 reversed the inhibitory effect of HMGA2 overexpression on EMT of gastric cancer cells and the expression of the stemness markers CD44 and OCT4 (Fig. 5A and B). The overexpression of HMGA2 significantly promoted EMT in SGC-7901 cells and the expression of the stemness markers CD44 and OCT4. However, the interference of TWIST1 expression also reversed the promoter effect of HMGA2 overexpression on EMT in gastric cancer cells and the expression of the stemness markers CD44 and OCT4 (Fig. 5A and B).

\section{Discussion}

HMGA2 plays an important role in EMT and in cancer stem cells (CSCs), but the mechanism by which HMGA2 stimulates downstream target genes to induce EMT and the acquisition of tumor stem cell properties remain unclear. The analysis of downstream regulatory networks of HMGA2 may be an important clue in the exploration of the correlation between EMT and CSCs.

We previously reported that HMGA2 can bind to the TWIST1 promoter region in gastric cancer (13). Therefore, an important regulatory relationship exists between HMGA2 and TWIST1. First, we detected the expression of HMGA2 and TWIST1 in pathological specimens and found that the immunohistochemical and protein expression of HMGA2 and TWIST1 in gastric cancer cells were high and showed a positive correlation compared with that of matched normal adjacent tissues. Furthermore, we detected the correlation between the protein expression of HMGA2 and TWIST1 in gastric epithelial cell lines and found that HMGA2 and TWIST1 were highly expressed in MKN-45 cells and were lowly expressed in SGC-7901 and MKN-28 cells, which was consistent with what has been observed in gastric cancer. To investigate the changes in TWIST1 after its expression was blocked and after the HMGA2 gene was overexpressed, we abrogated HMGA2 expression in MKN-45 cells and overexpressed HMGA2 in SGC-7901 cells. We found that the TWIST1 protein expression level was correspondingly increased and decreased, respectively, in MKN-45 and SGC-7901 cells. To further confirm the regulatory effect of HMGA2 on TWIST1, we constructed a reporter vector of TWIST1 and found that HMGA2 significantly improved the promoter activity of TWIST1. Therefore, both our preliminary results and this experimental result indicate that TWIST1 is a direct downstream target gene of HMGA2.

Previous studies have shown that the overexpression of HMGA 2 can significantly promote the invasion and migration of gastric cancer cells (27), whereas TWIST1 overexpression was also found to promote migration and invasion in breast, esophageal and colorectal cancer (26,29-32). However, whether HMGA2 regulates migration and invasion in gastric cancer through TWIST1 has not been confirmed. First, we inhibited the expression of HMGA2 in MKN-45 cells and simultaneously overexpressed TWIST1; we also overexpressed HMGA2 in SGC-7901 cells and simultaneously inhibited 
A

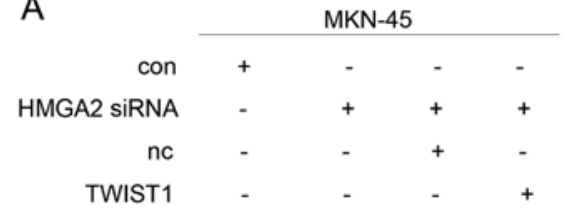

HMGA2

TWIST1

E-cadherin

$\mathrm{N}$-cadherin

CD44

OCT4

GAPDH

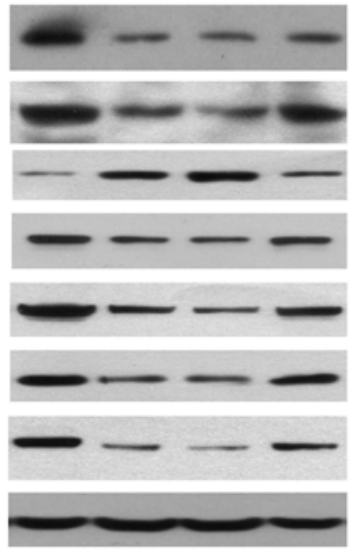

vimentin

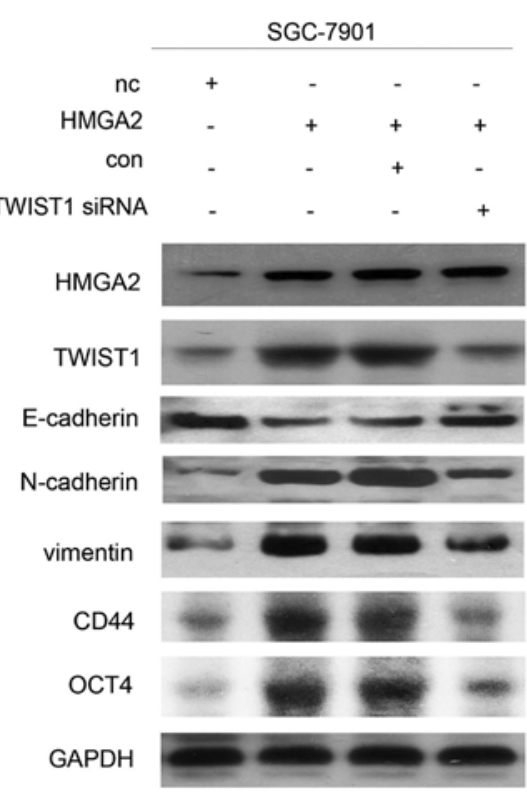

B
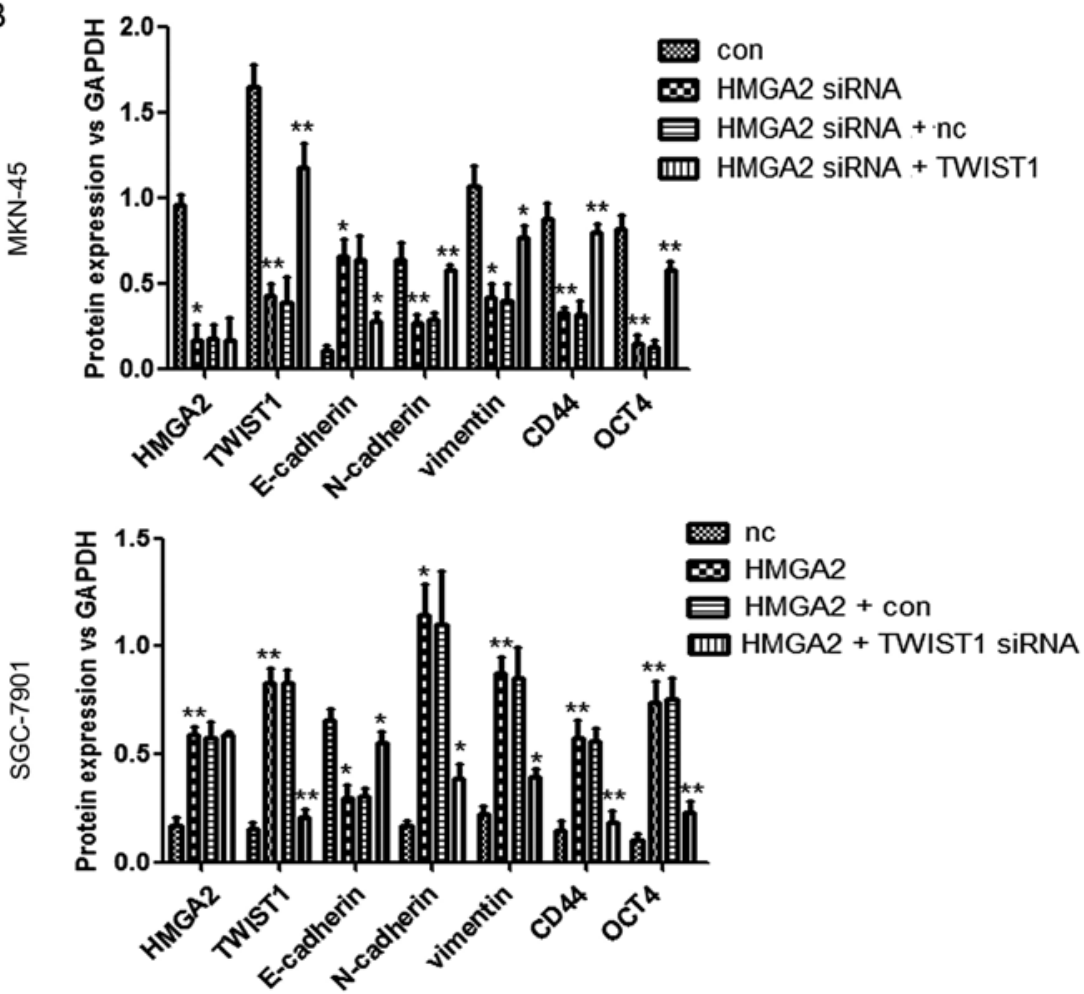

Figure 5. HMGA2 affects EMT of gastric cancer cells and the expression of stemness indicators via TWIST1. (A) MKN-45 cells were transfected with con, HMGA2 shRNA, HMGA2 shRNA + NC, or HMGA2 shRNA + TWIST1; and then EMT (E-cadherin, N-cadherin and vimentin expression) and the expression of stemness markers (CD44 and OCT4) in the cells were detected by western blot analysis assay in each group. SGC-7901 cells were transfected with NC, HMGA2, HMGA2 + con, or HMGA2 + TWIST1 shRNA; and then EMT (E-cadherin, N-cadherin and vimentin expression) and the expression of stemness markers (CD44 and OCT4) in the cells were detected by western blot analysis assay in each group. The experiment was repeated three times. (B) Quantification assays of each index of the western blot analysis results. ${ }^{*} \mathrm{P}<0.05,{ }^{* *} \mathrm{P}<0.01$. HMGA2, high mobility group protein $\mathrm{A} 2$.

the expression of TWIST1. We then observed the role of TWIST1 and HMGA2 on invasion and migration, initiation of EMT and acquisition of tumor stem cell properties in gastric cancer cells. The results showed that TWIST1 overexpression reversed the decrease in the invasion and migration abilities and EMT and the expression of stemness markers induced by HMGA2 interference in MKN-45 gastric cancer cells; the results also showed that TWIST1 interference inhibited the increase in HMGA2-mediated invasion and migration, EMT and the expression of stemness markers induced by HMGA2 overexpression in SGC-7901 gastric cancer cells. To further validate the effect of TWIST1 on the metastasis of gastric cancer cells, we performed in vivo tumor metastasis experiments in nude mice. In this model, the expression of HMGA2 was inhibited and TWIST1 was overexpressed in MKN-45 cells. We found that TWIST1 overexpression 
could reverse the inhibition of HMGA2 interference on the metastasis of MKN-45 cells.

In summary, HMGA2 can directly regulate the expression of TWIST1, which induces migration and invasion, EMT and the acquisition of tumor stem cell properties in gastric cancer cells.

\section{Acknowledgements}

This study was supported by grants from the National Natural Science Foundation of China (no. 81272753). The authors gratefully thank the Molecular Oncology and Epigenetics Laboratory of the First Affiliated Hospital of Chongqing Medical University for providing the clinical tissue specimens and the corresponding data.

\section{References}

1. Thiery JP, Acloque H, Huang RY and Nieto MA: Epithelialmesenchymal transitions in development and disease. Cell 139: 871-890, 2009.

2. Sethi S, Macoska J, Chen W and Sarkar FH: Molecular signature of epithelial-mesenchymal transition (EMT) in human prostate cancer bone metastasis. Am J Transl Res 3: 90-99, 2010.

3. Biddle A, Liang X, Gammon L, Fazil B, Harper LJ, Emich H, Costea DE and Mackenzie IC: Cancer stem cells in squamous cell carcinoma switch between two distinct phenotypes that are preferentially migratory or proliferative. Cancer Res 71: 5317-5326, 2011.

4. Chen C, Wei Y, Hummel M, Hoffmann TK, Gross M, Kaufmann AM and Albers AE: Evidence for epithelial-mesenchymal transition in cancer stem cells of head and neck squamous cell carcinoma. PLoS One 6: e16466, 2011.

5. Motoyama K, Inoue H, Nakamura Y, Uetake H, Sugihara $K$ and Mori M: Clinical significance of high mobility group A2 in human gastric cancer and its relationship to let-7 microRNA family. Clin Cancer Res 14: 2334-2340, 2008.

6. Belge G, Meyer A, Klemke M, Burchardt K, Stern C, Wosniok W, Loeschke S and Bullerdiek J: Upregulation of HMGA2 in thyroid carcinomas: A novel molecular marker to distinguish between benign and malignant follicular neoplasias. Genes Chromosomes Cancer 47: 56-63, 2008.

7. Huang ML, Chen CC and Chang LC: Gene expressions of $H M G I-C$ and $H M G I(Y)$ are associated with stage and metastasis in colorectal cancer. Int J Colorectal Dis 24: 1281-1286, 2009.

8. Fusco A and Fedele M: Roles of HMGA proteins in cancer. Nat Rev Cancer 7: 899-910, 2007.

9. Hock R, Furusawa T, Ueda T and Bustin M: HMG chromosomal proteins in development and disease. Trends Cell Biol 17: 72-79, 2007.

10. Wu J, Liu Z, Shao C, Gong Y, Hernando E, Lee P, Narita M, Muller W, Liu J and Wei JJ: HMGA2 overexpression-induced ovarian surface epithelial transformation is mediated through regulation of EMT genes. Cancer Res 71: 349-359, 2011.

11. Chaw SY, Majeed AA, Dalley AJ, Chan A, Stein S and Farah CS: Epithelial to mesenchymal transition (EMT) biomarkers - E-cadherin, beta-catenin, APC and vimentin - in oral squamous cell carcinogenesis and transformation. Oral Oncol 48: 997-1006, 2012.

12. Nishino J, Kim I, Chada K and Morrison SJ: Hmga2 promotes neural stem cell self-renewal in young but not old mice by reducing p16 ${ }^{\text {Ink4a }}$ and p19 ${ }^{\text {Arf }}$ expression. Cell 135: 227-239, 2008.

13. Zha L, Wang Z, Tang W, Zhang N, Liao G and Huang Z: Genome-wide analysis of HMGA2 transcription factor binding sites by ChIP on chip in gastric carcinoma cells. Mol Cell Biochem 364: 243-251, 2012.

14. Dave B, Mittal V, Tan NM and Chang JC: Epithelial-mesenchymal transition, cancer stem cells and treatment resistance. Breast Cancer Res 14: 202, 2012.
15. Copley MR, Babovic S, Benz C, Knapp DJ, Beer PA, Kent DG, Wohrer S, Treloar DQ, Day C, Rowe K, et al: The Lin28b-let-7-Hmga2 axis determines the higher self-renewal potential of fetal haematopoietic stem cells. Nat Cell Biol 15: 916-925, 2013.

16. Sun M, Gomes S, Chen P, Frankenberger CA, Sankarasharma D, Chung $\mathrm{CH}$, Chada KK and Rosner MR: RKIP and HMGA2 regulate breast tumor survival and metastasis through lysyl oxidase and syndecan-2. Oncogene 33: 3528-3537, 2014.

17. Kong D, Su G, Zha L, Zhang H, Xiang J, Xu W, Tang Y and Wang Z: Coexpression of HMGA2 and Oct4 predicts an unfavorable prognosis in human gastric cancer. Med Oncol 31: 130, 2014.

18. Yang J, Mani SA, Donaher JL, Ramaswamy S, Itzykson RA, Come C, Savagner P, Gitelman I, Richardson A and Weinberg RA: Twist, a master regulator of morphogenesis, plays an essential role in tumor metastasis. Cell 117: 927-939, 2004.

19. Eckert MA, Lwin TM, Chang AT, Kim J, Danis E, OhnoMachado L and Yang J: Twist1-induced invadopodia formation promotes tumor metastasis. Cancer Cell 19: 372-386, 2011.

20. Pozharskaya V, Torres-González E, Rojas M, Gal A, Amin M, Dollard S, Roman J, Stecenko AA and Mora AL: Twist: A regulator of epithelial-mesenchymal transition in lung fibrosis. PLoS One 4: e7559, 2009.

21. Yu L, Li HZ, Lu SM, Tian JJ, Ma JK, Wang HB and Xu W: Down-regulation of TWIST decreases migration and invasion of laryngeal carcinoma Hep- 2 cells by regulating the E-cadherin, N-cadherin expression. J Cancer Res Clin Oncol 137: 1487-1493, 2011.

22. Mani SA, Guo W, Liao MJ, Eaton EN, Ayyanan A, Zhou AY, Brooks M, Reinhard F, Zhang CC, Shipitsin M, et al: The epithelial-mesenchymal transition generates cells with properties of stem cells. Cell 133: 704-715, 2008.

23. Yang WH, Lan HY, Huang CH, Tai SK, Tzeng CH, Kao SY, Wu KJ, Hung MC and Yang MH: RAC1 activation mediates Twist1-induced cancer cell migration. Nat Cell Biol 14: 366-374, 2012.

24. Yang MH, Hsu DS, Wang HW, Wang HJ, Lan HY, Yang WH, Huang CH, Kao SY, Tzeng CH, Tai SK, et al: Bmil is essential in Twist1-induced epithelial-mesenchymal transition. Nat Cell Biol 12: 982-992, 2010.

25. Sung CO, Lee KW, Han S and Kim SH: Twist1 is up-regulated in gastric cancer-associated fibroblasts with poor clinical outcomes. Am J Pathol 179: 1827-1838, 2011.

26. Valdés-Mora F, Gómez del Pulgar T, Bandrés E, Cejas P, Ramírez de Molina A, Pérez-Palacios R, Gallego-Ortega D, García-Cabezas MA, Casado E, Larrauri J, et al: TWIST1 overexpression is associated with nodal invasion and male sex in primary colorectal cancer. Ann Surg Oncol 16: 78-87, 2009.

27. Zha L, Zhang J, Tang W, Zhang N, He M, Guo Y and Wang Z: HMGA2 elicits EMT by activating the Wnt/ $\beta$-catenin pathway in gastric cancer. Dig Dis Sci 58: 724-733, 2013.

28. Xu MZ, Yao TJ, Lee NP, Ng IO, Chan YT, Zender L, Lowe SW, Poon RT and Luk JM: Yes-associated protein is an independent prognostic marker in hepatocellular carcinoma. Cancer 115: 4576-4585, 2009.

29. Mani SA, Yang J, Brooks M, Schwaninger G, Zhou A, Miura N, Kutok JL, Hartwell K, Richardson AL and Weinberg RA: Mesenchyme Forkhead 1 (FOXC2) plays a key role in metastasis and is associated with aggressive basal-like breast cancers. Proc Natl Acad Sci USA 104: 10069-10074, 2007.

30. Cheng GZ, Chan J, Wang Q, Zhang W, Sun CD and Wang LH: Twist transcriptionally up-regulates AKT2 in breast cancer cells leading to increased migration, invasion, and resistance to paclitaxel. Cancer Res 67: 1979-1987, 2007.

31. Ai L, Kim WJ, Alpay M, Tang M, Pardo CE, Hatakeyama S, May WS, Kladde MP, Heldermon CD, Siegel EM, et al: TRIM29 suppresses TWIST1 and invasive breast cancer behavior. Cancer Res 74: 4875-4887, 2014.

32. Lee KW, Sung CO, Kim JH, Kang M, Yoo HY, Kim HH, Um SH and Kim SH: CD10 expression is enhanced by Twist1 and associated with poor prognosis in esophageal squamous cell carcinoma with facilitating tumorigenicity in vitro and in vivo. Int J Cancer 136: 310-321, 2015. 